\title{
Minimal-invasiver Blutzuckersensor (MIBS)
}

\author{
M. Birkholz ${ }^{*}$, K.-E. Ehwald, M. Fröhlich, P. Kulse, T. Basmer, R. Ehwald ${ }^{1}$, T. Guschauski ${ }^{1}$, \\ U. Stoll ${ }^{2}$, H. Siegel ${ }^{2}$, S. Schmaderer ${ }^{3}$, J. Szeponik ${ }^{3}$, D. Zahn ${ }^{3}$ \\ IHP - Leibniz-Institut für innovative Mikroelektronik, Im Technologiepark 25, 15236 Frankfurt (Oder) \\ ${ }^{1}$ Humboldt-Universität zu Berlin, Institut für Biologie, Invalidenstr. 42, 10115 Berlin \\ ${ }^{2}$ Sitec GmbH, Friedrich-Franz-Str. 19, 14770 Brandenburg \\ ${ }^{3}$ BST Bio Sensor Technology GmbH, Buchholzer Sr. 55-61, 13156 Berlin \\ *birkholz@ihp-microelectronics.com
}

\section{Kurzfassung}

Ein minimal-invasiver Glucose-Affinitätssensor für den transdermalen Einsatz wird beschrieben. Die Signalbildung beruht auf der von der Glucosekonzentration abhängigen Viskosität einer sensitiven Flüssigkeit, in der Dextranmoleküle durch das tetravalente Lektin Concanavalin A reversibel vernetzt sind. Die Viskositätsmessung erfolgt in einer mit der sensitiven Flüssigkeit gefüllten Mikrodialysekammer durch ein voll integriertes mikroelektromechanisches System (MEMS). Als Viskositätsmaß dient die Zeit, die für die definierte Verbiegung eines aus Titannitrid bestehenden $50 \mathrm{~nm}$ starken Kantilevers unter dem Einfluss einer HF-Spannung benötigt wird. Diese Zeit ist von der Glucosekonzentration in ihrem medizinisch relevanten Bereich reversibel abhängig. Die Polymere der sensitiven Flüssigkeit sind vom Milieu durch eine $60 \mu \mathrm{m}$ dicke Kompositmembran getrennt. Ihre poröse keramische Struktur sichert die Konstanz des eingeschlossenen Flüssigkeitsvolumens und erleichtert die Verbindung der Membran mit der Sonde. Die ca. $3 \mu \mathrm{m}$ dicke semipermeable organische Trennschicht der Kompositmembran ermöglicht einen schnellen Glucoseaustausch und stellt eine langzeitstabile Barriere für Proteine mit einem Molekulargewicht oberhalb von $5 \mathrm{kDa}$ dar. In vitro Untersuchungen zeigen eine hohe Messgenauigkeit und eine hohe Kennlinienstabilität des Sensors.

\begin{abstract}
A minimally invasive glucose affinity sensor for transdermal application is described. Signalling is based on viscosity measurement in a sensitive liquid, wherein dextran molecules of high moleclular weight are reversibly cross-linked by tetravalent lectin concanavalin $A$. The viscosity measurement is carried out in a microdialysis chamber filled with the sensitive liquid by a fully embedded microelectromechanical system (MEMS). The viscosity parameter determined by the MEMS is the time required for a defined deflection of a $50 \mathrm{~nm}$ thick titanium nitride cantilever by a HF voltage. This time is reversibly dependend on the glucose concentration in its medically significant range. The polymers of the sensitive liquid are separated from the milieu by a $60 \mu \mathrm{m}$ thick composite membrane. Its porous ceramic structure ensures a defined volume of the enclosed liquid and facilitates its connection with the silicon probe. The ca. $3 \mu \mathrm{m}$ thick semipermeable organic separation layer of the composite membrane enables rapid glucose exchange and forms a long term stable barrier for proteins with a molecular weight above $5 \mathrm{kDa}$. In vitro studies showed a high measuring accuracy and stability of the sensors' response curve.
\end{abstract}




\section{Einführung}

Die Erfassung der für Diabetiker typischen Schwankungen des Blutzuckergehaltes ist die Grundlage für eine optimale Therapie durch Insulingaben. Der große Bedarf an Sensoren für optimales Glucosemonitoring und die hohen Anforderungen, die aus medizinischer Sicht an ihre Zuverlässigkeit und Praktikabilität gestellt werden müssen, stellen seit langem eine große Herausforderung für die Medizintechnik dar. Messungen des Blutzuckerspiegels erfolgen heute überwiegend mit Enzymsensoren auf der Basis von Einweg-Teststreifen [1,2]. Da hierzu eine psychologische Barriere für die hiermit verbundene kleine Selbstverletzung besteht [3], werden die Messungen zu selten durchgeführt, wodurch eine vorbeugende Therapie gegen die Spätfolgen der hyperglykämischen Zustände beeinträchtigt wird [3]. Von großem medizinischem und kommerziellem Interesse sind beim gegenwärtigen Stand der Entwicklung miniaturisierte transdermale Sensoren für das kontinuierliche Glucosemonitoring, die in minimal-invasive Sonden integriert sind [5]. Trotz beachtlicher Fortschritte wurden bisher noch keine voll befriedigenden Ergebnisse mit solchen Glucose-Sensoren erzielt. Amperometrische Sensoren dieses Typs, die zur Signalwandlung an einer miniaturisierten Elektrode das Enzym Glucoseoxidase nutzen und das Reaktionsprodukt Wasserstoffperoxid detektieren, bleiben einige Tage funktionsfähig und werden kommerziell für diagnostische Zwecke angeboten. Sie zeigen jedoch im Unterhautgewebe Veränderungen ihrer Empfindlichkeit und erfordern daher regelmäßiges Nachkalibrieren [6]. Als mögliche Ursachen kommen u. a. der schwer vermeidbare Einfluss des Gewebestoffwechsels auf die Konzentration des Wasserstoffperoxids oder die Elektrodenreaktion in Betracht [7].

Neben der Verbesserung der amperometrischen Enzymsensoren gab es im vergangenen Jahrzehnt Fortschritte bei der Entwicklung von Affinitätssensoren, bei denen die Messgröße durch die reversible Bindung der Glucose an Affinitätsbindungsorte eines Rezeptormoleküls moduliert wird [8]. Der im Folgenden beschriebene minimal-invasive Glucosesensor beruht auf dem viskosimetrischen Messprinzip $[9,10]$. Er nutzt das Protein Concanavalin A (ConA) als Rezeptormolekül in einer sensitiven Flüssigkeit, in der hochmolekulare Dextranmoleküle mit einem Moleküldurchmesser von ca. $80 \mathrm{~nm}$ durch reversible Affinitätsbindungen zu einem flüssigen Netzwerk verbunden sind. Das ConA liegt bei physiologischen pHWerten als Tetramer vor. Jedes Monomer verfügt über eine Bindungstelle, die in der sensitiven Flüssigkeit mit freien Glucosemolekülen oder den Glucose-Endgliedern der Dextranmoleküle besetzt ist. Da die relativ schwache Affinitätsbindung eines Glycoliganden am ConA nur sehr kurze Zeit beständig ist (Mikrosekundenbereich), findet ein ständiger Ligandenaustausch statt, bei dem die Glucosemoleküle mit den terminalen Glucoseresten der verwzeigten Dextranmoleküle konkurrieren. Die Erhöhung der Glucosekonzentration führt zu einer Lockerung, ihre Erniedrigung zur Stabilisierung des makromolekularen Netzwerkes. Die bei ausreichender Schergeschwindigkeit gemessene Viskosität der sensitiven Flüssigkeiten ist von der Glucosekonzentration daher in definierter Weise abhängig [10].

Mit einem kontinuierlich arbeitenden transdermalen viskosimetrischen Glucosesensor, der den Strömungswiderstand der sensitiven Flüssigkeit nach dem Durchlaufen einer implantierten Mikrodialysefaser erfasst, konnte die Glucosekonzentration im humanen subkutanen Gewebe nahezu driftfrei im Verlauf mehrerer Tage gemessen werden [12,13]. Seiner kostengünstigen industriellen Produktion und seiner Anwendung zur Selbstkontrolle des Blutzuckerspiegels steht allerdings das recht aufwändige mikrofluidische System entgegen. Im Folgenden wird ein neuer viskosimetrischer Affinitätssensor für den transdermalen Einsatz vorgestellt, bei dem das Viskosimeter als voll integriertes mikroelektromechanisches System (MEMS) vorliegt. Es wird in einer sehr kleinen Menge der sensitiven Flüssigkeit eingesetzt, die in eine Mikrodialysekammer eingeschlossen ist [14,15]. Das miniaturisierte Mess-System kann in eine minimalinvasive Sonde für die transdermale Anwendung integriert werden. An der Realisierung dieses Konzepts arbeiten seit dem Jahre 2006 Arbeitsgruppen des IHP, der HU Berlin und 
der mittelständischen Unternehmen Sitec und BST. Die wichtigsten Ziele der von diesem Konsortium bearbeiteten Verbundprojekte waren:

- Entwicklung eines voll integrierten mikroelektromechanischen Systems (MEMS), das im Elektrolytmilieu der interstitiellen Flüssigkeit langzeitstabil genaue Viskositätsmessungen der sensitiven Flüssigkeit ermöglicht,

- Entwicklung einer für Proteine und polymere Glykoliganden undurchlässigen Membran zur Begrenzung der Mikrodialysekammer, die sich durch hohe Glucosepermeabilität, konstante Fläche und günstige verbindungstechnische Eigenschaften auszeichnet,

- Optimierung der Zusammensetzung der sensitiven Flüssigkeit in Bezug auf die Glucosekennlinien des Mikroviskosimeters im Bereich von 0 bis 25 mM,

- Entwicklung einer Prinziplösung zum Einbringen der sensitiven Flüssigkeit in die miniaturisierte Mikrodialysekammer,

- Entwicklung einer Prinziplösung zur Gestaltung der transdermalen Sonde,

- Entwicklung einer Prinziplösung für die Datenübertragung und Datenverarbeitung,

- Dokumentation der Sensorfunktion in vitro,

- Schaffung der Voraussetzungen für transdermale Messungen in klinischen Studien (Miniaturisierung, Sterilisation und Konservierung).

\section{Das mikroelektromechanische System}

Ein wesentliches Bauelement des Sensors stellt das mikroelektromechanische System (MEMS) dar, mit dem die Viskosität der sensorischen Flüssigkeit bestimmt und in ein elektrisches Signal transformiert wird. Dieses MEMS wird in einer 0,25 $\mu \mathrm{m}$ BiCMOS-Technologie des IHP hergestellt und stellt eines der ersten in eine mikroelektronische Schaltung voll integriertes MEMS zur Untersuchung von Flüssigkeiten in biologischen Organismen dar. Der Chip hat Abmessungen von nur 0,4 × 1,3 ×0,2 mm. Für die Viskositätsmessung wird ein Aktuatorelement verwendet, das als 4-seitig eingespannter Bügel ausgeführt ist [16] und sich im Abstand von 2,5 $\mu \mathrm{m}$ über einer Grundplatte befindet (Abb. 1). Beim Anlegen einer HFSpannung wird der elastisch auslenkbare Bügel in Richtung zur Grundplatte verbogen, so dass sich die elektrische Kapazität der Anordnung verändert. Messsignal ist die Zeit, die vom Anlegen der Spannung bis zur Auslenkung des Bügels zu einer definierten Position benötigt wird, bei der ein festgelegter Wert der Kapazitätsänderung erreicht ist. Mit Hilfe einer an die mikromechanischen Erfordernisse angepassten integrierten HF-Schaltung wird nach dieser Zeit die HF-Spannung abgeschaltet [17]. Diese Zeit, die Schaltzeit $t_{s w}$, dient als Messgröße für die Viskosität.

Aufgrund des direkten Kontakts des MEMS mit den Elektrolyten der interstitiellen Flüssigkeit war besonderer Wert auf eine korrosionsbeständige Ausführung der exponierten Komponenten zu legen. Die Grundplatte und der mechanisch auslenkbare Bügel werden aus diesem Grund aus Titannitrid (TiN) gefertigt, das eine elektrische Leitfähigkeit aufweist, die mit der von Metallen vergleichbar ist. TiN ist ein in der Halbleiterfertigung etabliertes Material und besitzt von allen im IHP-Reinraum verfügbaren Metallen die höchste Korrosionsbeständigkeit gegenüber Körperflüssigkeiten [18]. In einem Freiwilligenversuch, bei dem der Sensorchip für drei Monate in subkutanes Gewebe implantiert wurde, war nach Explantation 


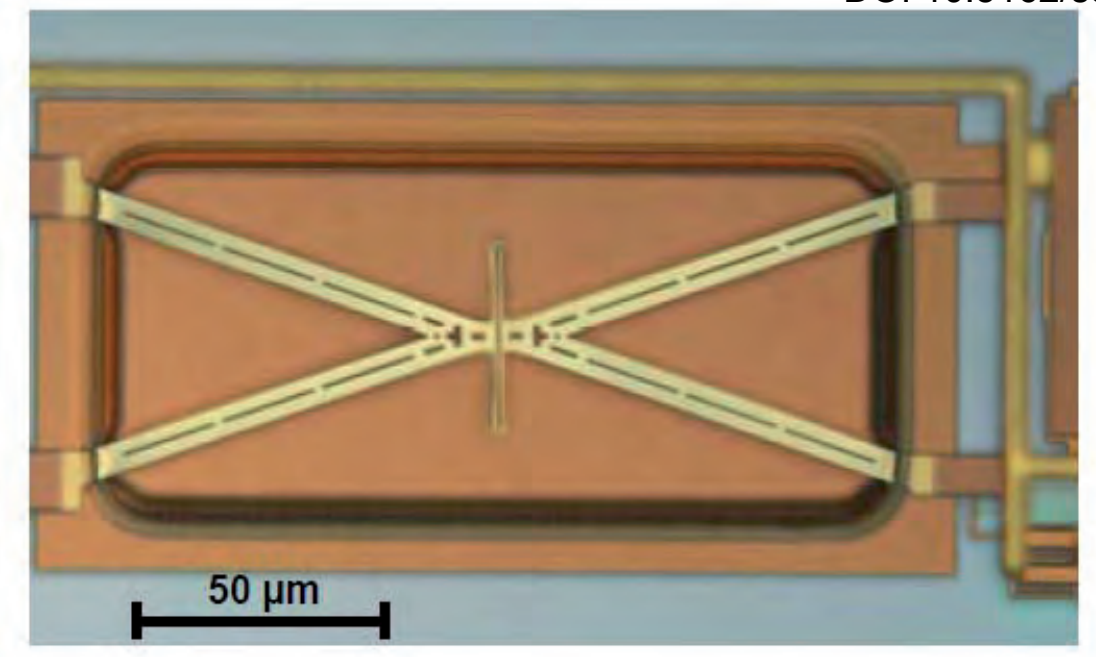

Abb. 1:

Mikroskopaufsicht auf den Titannitrid-Bügel des MEMS. In der Mitte befindet sich ein Dehnelement, das eine hohe Stabilität der Bewegung bei geringer Effektivspannung gewährleistet.

kein messbarer Abbau des TiN festzustellen; auch die umgebenden Dünnschichten aus $\mathrm{SiO}_{2}$ und Passivierungsnitrid SiON waren nur sehr geringen Abbauraten in der Größenordnung von $50 \mathrm{~nm} /$ Monat ausgesetzt [19]. Aufgrund dieser Untersuchungen ist davon auszugehen, dass die mikroelektronischen und mikromechanischen Komponenten des Sensorchips durch ihren Einsatz im Gewebe im Verlauf von 1-2 Wochen in ihrer Funktion nicht beeinträchtigt werden.

\section{Integration des Sensorchips und der Mikrodialysekammer in den Sondenkörper}

Die Sensorsonde war so aufzubauen, dass alle elektrischen Leitungen hermetisch gegen die Elektrolytlösungen abgeschirmt werden. Zudem waren Materialien zu verwenden, die im Rahmen des Medizinproduktegesetzes bereits zugelassen sind, so dass sie in klinischen Studien ohne umfangreiche Vorläuferstudien verwendet werden können. Es wurde eine aufbau- und verbindungstechnische Prozesskette entwickelt, mit der der Sensorchip in eine patiententaugliche Sonde integriert werden kann [20]. Wichtige Teilschritte sind die Freiätzung der mikromechanischen Kavitäten und Aktuatorbügel [21] sowie die nachfolgende kritische Punkttrocknung mit überkritischem $\mathrm{CO}_{2}$ [22], die lasergestützte Vereinzelung der Chips [23], die Verbindung des Sensorchips mit einer als starrflexiblen Leiterplatte ausgeführten Zuleitung durch flip chip bonding sowie die Integration der Sensorchips samt Flexleitung in den Sondenkörper. Letzteres erfordert die Gewährleistung einer ausreichenden Wärmeableitung, da in einem einzelnen Messzyklus von rund $100 \mathrm{~ms}$ im Schnitt $100 \mathrm{~mW}$ thermische Leistung generiert werden. 


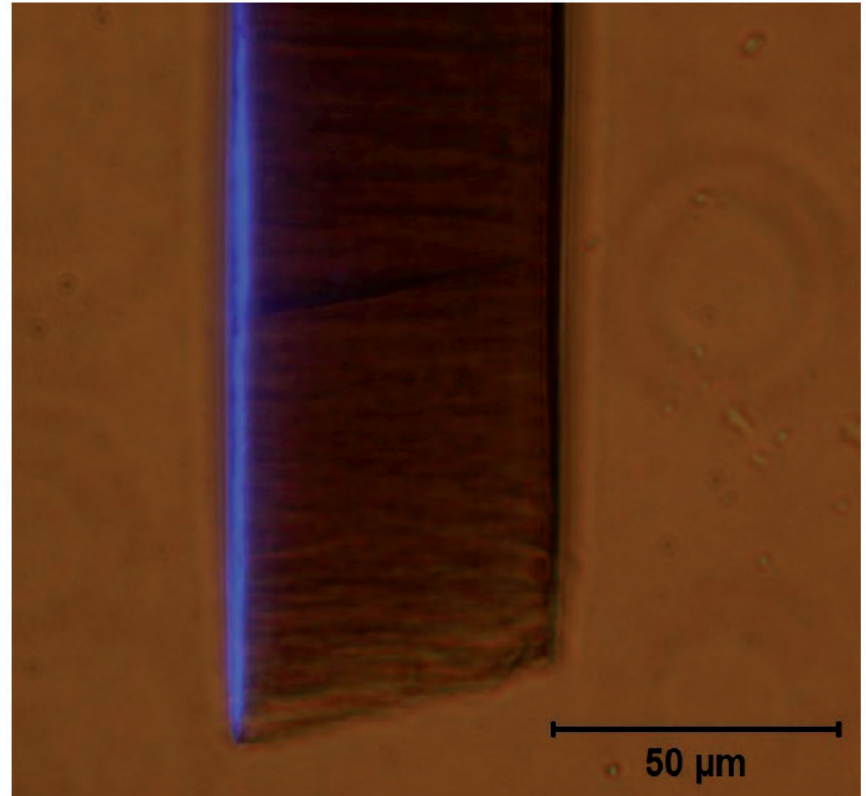

Abb. 2.

Foto eines Schnittes durch die Kompositmembran. In die poröse keramische Stützschicht ist einseitig eine organische Trennschicht integriert, die mit einem Fluoreszenzmarker sichtbar wird.

Zur Gestaltung des Interface zwischen der sensitiven Flüssigkeit und der zu analysierenden Körperflüssigkeit wurde eine semipermeable Membran [24] entwickelt, die ein Komposit aus einer $60 \mu \mathrm{m}$ dicken hochporösen keramischen Stützschicht und einer einseitig in deren Poren verankerten etwa $3 \mu \mathrm{m}$ dicken semipermeablen organischen Trennschicht darstellt (Abb. 2). Die von der Firma BST hergestellte Membran zeichnet sich durch eine hohe Permeabilität für Glucose (ca. $1 \mu \mathrm{m} \mathrm{s}^{-1}$ ) aus und besitzt im Langzeittest eine sehr niedrige und scharfe Größenausschlussgrenze (Tab. 1).

Tabelle 1.

Größentrenngrenze verschiedener Chargen der zur Dialyse eingesetzten Kompositmembran.

Charge
1
1
2
3
4
5

Diffusionszeit $[\mathrm{d}]$
6
14
14
6
6
6

Größentrenngrenze [nm]
1,4
1,9
1,8
1,7
1,7
1,7

* Die Größentrenngrenze ist der chromatographisch ermittelte Stokes-Durchmesser der Molekülgrößenfraktion des Testpolymeren [25], deren Menge im Retentat nach der angegebenen Diffusionszeit $98 \%$ der eingesetzten Menge beträgt. Polypeptide mit einer Molmasse von 5 kDa besitzen einen Stokesschen Durchmesser von ca. 2,2 $\mathrm{nm}$ [26].

Die Ausstattung der Dialysekammer mit der Kompositmembran ermöglicht die Integration eines sehr kleinen Volumens der sensitiven Flüssigkeit in die Sonde (Abb. 3). Da die Stützschicht der Kompositmembran aus einem hochporösen, rigiden keramischen Material besteht, ist ihre Fläche keinen 
Veränderungen durch Quellung oder Dehnung unterworfen. Dadurch ist es möglich, in der Dialysekammer ein sehr kleines Volumen (ca. $100 \mathrm{nl}$ ) bei einer verhältnismäßig großen Oberfläche der semipermeablen Membran (ca. $0.5 \mathrm{~mm}^{2}$ ) konstant $\mathrm{zu}$ halten. Das ist für die Stabilität des Messparameters besonders wichtig, weil die Viskosität der sensitiven Flüssigkeit eine starke Abhängigkeit von der Konzentration der sensorischen Polymere besitzt. Ein weiterer wichtiger Vorteil der verwendeten Membran besteht darin, dass sie sich gut mit dem Sondenkörper verbinden lässt.

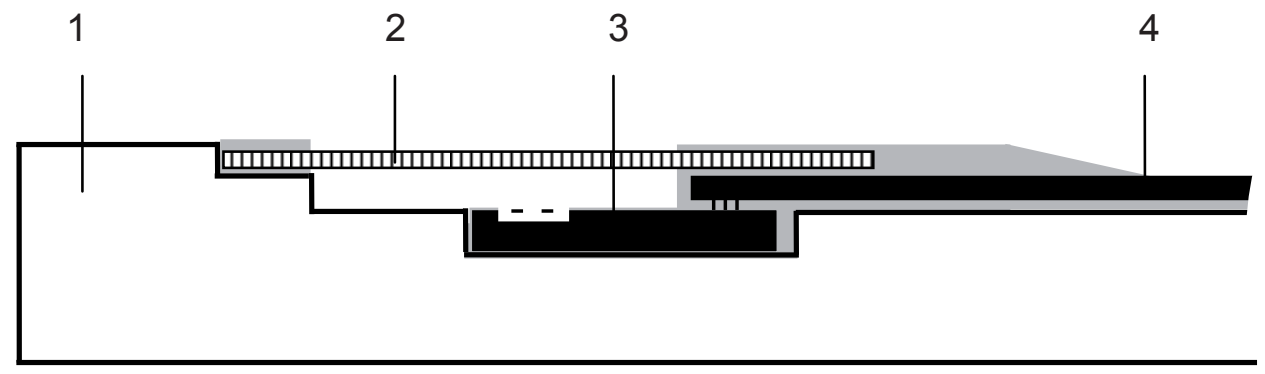

\begin{abstract}
Abb.3.
Längsschnitt durch den vorderen Teil der transdermalen Sonde (schematisch). Der MEMS-Chip ist in den gut wärmeleitenden Sondenkörper (1) integriert. Das Lumen der Dialysekammer mit der sensitiven Flüssigkeit wird durch die Kompositmembran (2) und durch das Mikroviskosimeter (3) begrenzt. Die Dicke der Flüssigkeitsschicht beträgt etwa $200 \mu$ m. Die elektrischen Kontakte zwischen der Zuleitung (4) und dem Sensorchip sind in einen inerten Kunststoff eingebettet.
\end{abstract}

Die Konzentrationen der Biopolymere in der sensorischen Flüssigkeit wurden an den am Aktuator entstehenden Scherdruck so angepasst, dass Änderungen der Glucosekonzentration im medizinisch relevanten Bereich (0-20 mM) ausreichend starke Veränderungen der viskositätsabhängigen Schaltzeit hervorrufen.

\title{
4. Aufbau des Gesamtsystems
}

Der Gesamtaufbau des Sensorsystems wurde so gestaltet, dass der in die Sonde integrierte Sensorchip in das Unterhautfettgewebe eingestochen werden kann und dort über eine intendierte Liegezeit von mehreren Tagen verbleibt, s. Abb. 4. Der Sensorchip ist über eine dreiadrige Leitung mit einem von der Fa. Sitec entwickelten Systemboard verbunden, dass sich in einem auf der Haut des Patienten angebrachten Transponder befindet und mit einem Mikrokontroller (TI MSP430) zur Messdatenauswertung sowie der Energieversorgung und Komponenten zur drahtlosen Datenübertragung $(2,45 \mathrm{GHz}-\mathrm{Band}$, ZigBee) bestückt ist. Die Messdaten werden an eine periphere Empfangseinheit (Laptop, Handy, Smartphone etc.) übermittelt. Sensorchip und Systemboard können über handelsübliche Akkus mit Ausgangsspannungen von 3,5 V (nominell) versorgt werden. Der Strombedarf des Sensorchips liegt während der Messung bei rund $30 \mathrm{~mA}$, so dass sich der Energieverbrauch einer Einzelmessung bei einer mittleren Messdauer von $200 \mathrm{~ms}$ mit rund $6 \mathrm{~mA} \cdot \mathrm{s}$ angeben lässt. Bei einem Einsatzszenario von einer Messung alle 2 min werden also 720*6/3600 mA.h pro Tag benötigt. In der gleichen Größenordnung liegt der Energieverbrauch des Systemboards, bei dem der Mikrokontroller und das Radiomodul die Hauptenergieverbraucher darstellen. Mit einer Akkukapazität von rund $100 \mathrm{~mA} \cdot \mathrm{h}$ lässt sich das Sensorsystem somit über einen Zeitraum von mehreren Tagen sicher betreiben. 


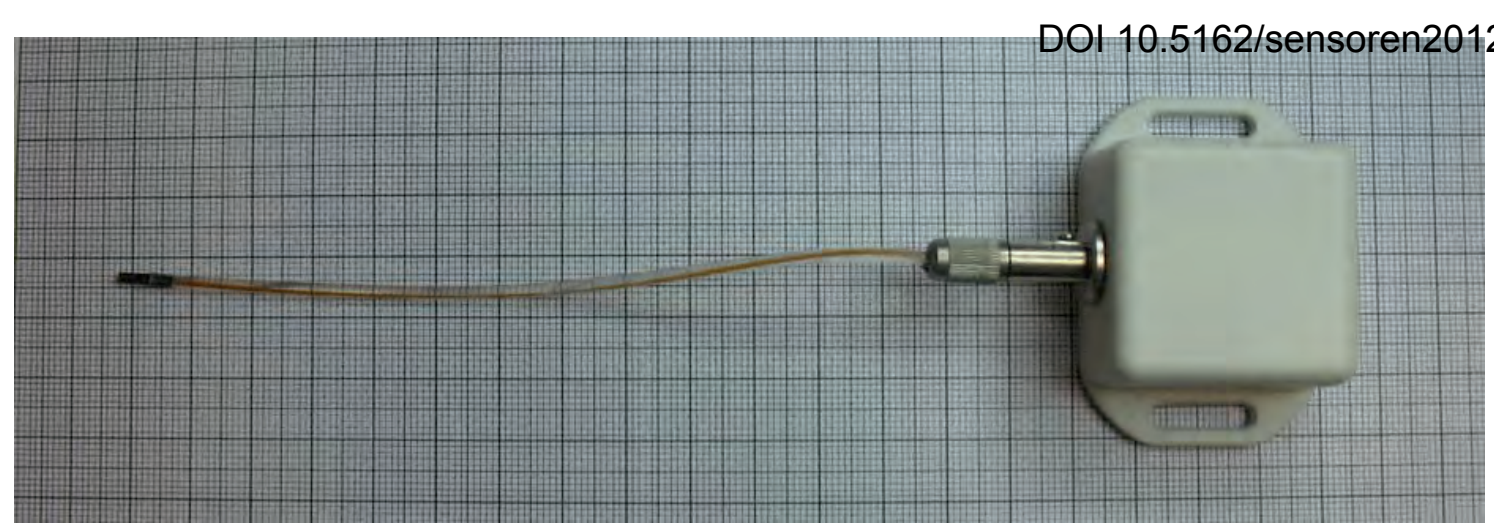

Abb. 4

Ansicht des Sensorsystems mit implantierbarer Sensor-Sonde (links) und dem Systemboard (rechte Box). Die Messdaten werden im Transponder gespeichert und können auf Anfrage drahtlos übermittelt werden. Die starflexible Leiterplatte stellt die Verbindung zwischen dem Sensorchip und dem Systemboard über eine einrastende Steckverbindung her.

\section{Messergebnisse und Diskussion}

Die aufgebauten Sensorsysteme wurden in Testlösungen untersucht, zu deren Herstellung auf $\mathrm{pH}=7,4$ gepufferte, isotone Elektrolytlösungen mit unterschiedlichen Glucosemengen versetzt wurden. Abb. 5 zeigt die Reaktion zweier Sensoren mit unterschiedlich eingestellter MEMS. Die relativ kurze Zeit, die für die Einstellung eines neuen Messwertes nach dem Wechsel des Mediums erforderlich war, beruht auf der diffusionskontrollierten Angleichung der Glucosekonzentrationen in der Dialysekammer an diejenige des Mediums. Die hierdurch verursachte Verzögerung des Messsignals beträgt wenige Minuten, was aus medizinisch-therapeutischer Sicht praktisch einer Echtzeit-Messung entspricht. Der Messfehler für die Glucosekonzentration liegt deutlich unter 5\%. Die instrumentelle Genauigkeit des Mikroviskosimeters ist wesentlich höher (nicht dargestellt). Leichte Veränderungen des Sensorsignals in beide Richtungen, die nach der Einstellung des Diffusionsgleichgewichtes beobachtet wurden (Abb. 5), erklären sich vermutlich daraus, dass sich kleine Temperaturgradienten oder Glucose-Konzentrationsgradienten im Versuchsgefäß nicht vermeiden ließen. Die Abhängigkeit der Schaltzeit von der Glucosekonzentration entspricht derjenigen der Viskosität sensitiver Flüssigkeiten auf der Grundlage von ConA und Dextran [27].

Eine Besonderheit des verwendeten Polymersystems besteht darin, dass die Viskosität und damit der Messparameter $t_{s w}$ bei niedrigen Glucosekonzentrationen besonders empfindlich auf Konzentrationsänderungen reagieren. Aus medizinischer Sicht ist dies als Vorteil zu betrachten. Eine hohe Messgenauigkeit in suboptimaler Glucosekonzentrationen $(0-3 \mathrm{mM})$ ist wichtig, weil der Sensor eine Warnfunktion gegen Unterzuckerung besitzen soll, die als Folge einer Insulin-Überdosis auftreten kann [28]. Die hohe Stabilität der biochemischen Komponenten der sensitiven Flüssigkeit und ihr perfekter Einschluss in der Mikrodialysekammer werden dadurch dokumentiert, dass die glucoseabhängige Schaltzeit bei konstanter Glucosekonzentration und Temperatur über mehrere Wochen keine signifikante Änderung zeigte. Die Schaltzeit wird bei einer Absenkung der Temperatur von 35 auf $25^{\circ} \mathrm{C}$ in Abhängigkeit von der Glucosekonzentration um 40 bis 50\% erhöht. Diese Temperaturabhängigkeit entspricht etwa derjenigen, die von der glucoseabhängigen Viskosität der sensitiven Flüssigkeit bereits bekannt ist [27]. Obwohl die Temperatur des Sensors beim transdermalen Einsatz relativ konstant bleiben sollte, enthält die integrierte Schaltung des Mikroviskosimeters die Möglichkeit der Temperaturmessung in situ, so dass temperaturbedingte Messfehler korrigiert werden können. 

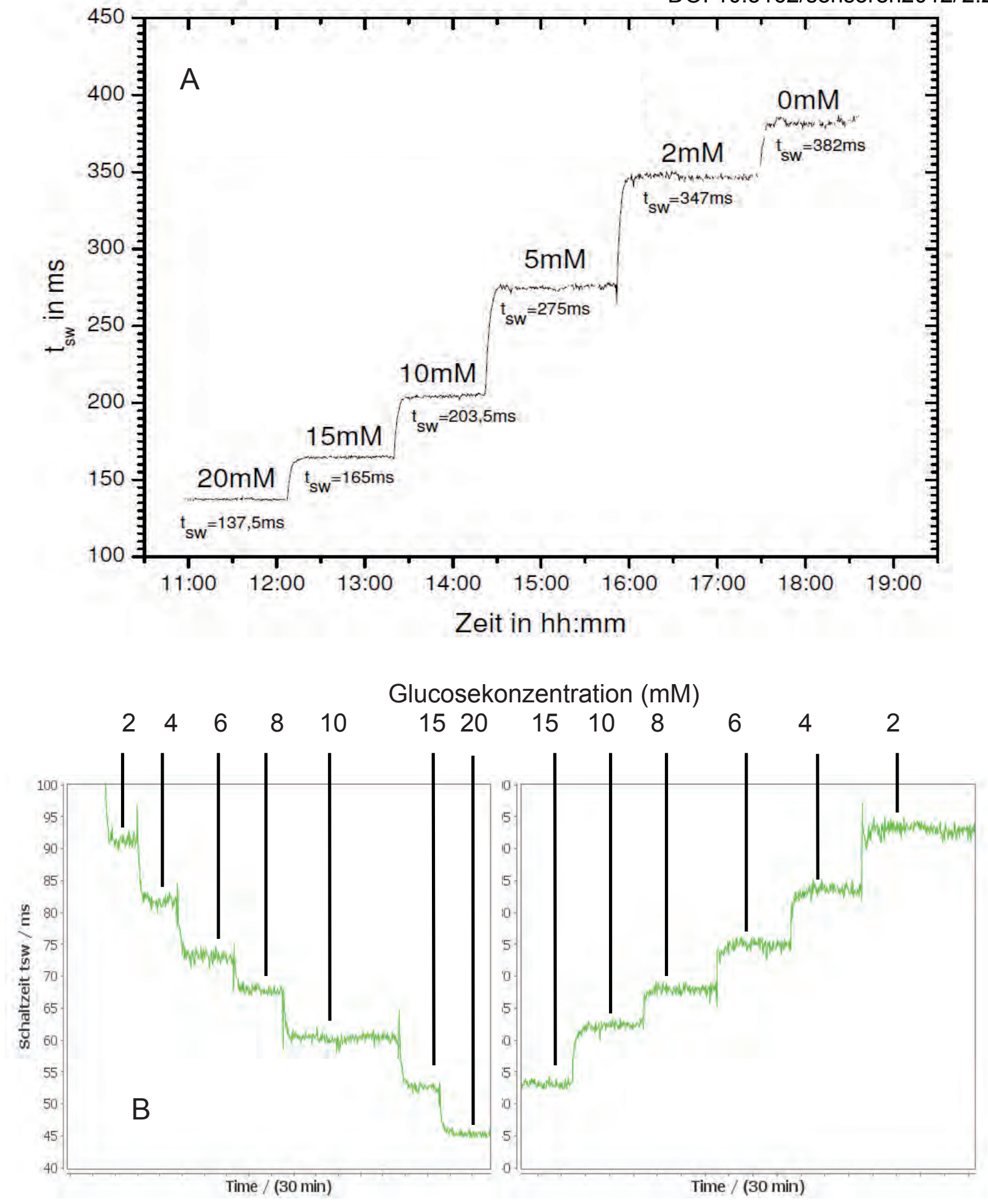

Abb. 5

Änderung der Schaltzeit $t_{s w}$ nach Veränderung der Glucosekonzentration des Standardelektrolytmediums bei $37^{\circ} \mathrm{C}$. A und B: Die Messkurven wurden mit zwei Sensoren aufgenommen, deren MEMS unterschiedlich eingestellt wurden. 
Der von uns vorgestellte viskosimetrische Affinitätssensor ist in die gegenwärtig breite Forschungs- und Entwicklungsarbeit auf dem Gebiet der implantierbaren Glucosesensoren einzuordnen [2] und soll daher abschließend mit ähnlichen neuen Entwicklungen auf diesem Gebiet verglichen werden. In letzter Zeit wurden auch von verschiedenen anderen Arbeitsgruppen Affinitätssensoren für das Gluosemonitoring entwickelt. Dabei wird angestrebt, die mit dem elektrochemischen Messprinzip der minimal-invasiven amperometrischen Sensoren verbundenen Schwierigkeiten zu vermeiden. Bei allen Affinitätssensoren wird das von Schultz et al. [8] vorgeschlagene Prinzip der Konkurrenz zwischen polymeren Affinitätsliganden und Glucose an einem Affinitätsrezeptor eingesetzt. Neben der Nutzung von ConA und anderen Lektinen werden in letzter Zeit zunehmend verzweigte Polymere mit zuckerbindenden BoronsäureSeitenketten als Rezeptoren für die Glucose verwendet [29]. Bisher wurde zur Signalwandlung vor allem die Abhängigkeit optischer oder mechanischer Eigenschaften der sensorischen Polymersysteme von der Glucosekonzentration genutzt. Es entstand eine größere Zahl von Affinitätssensoren für Glucose, in denen der einfache Fluoreszenzassay der ersten Prototypen [8] weiterentwickelt wurde [30-32] oder die Änderung des Brechungsindex des Polymersystems [31] zur Generation eines optischen Signals (Lichtstreuung) eingesetzt wird. Verschiedene dieser Sensoren wurden minimalinvasiv gestaltet [30, 32]. Ihre Anwendung im Unterhautfettgewebe erfordert jedoch das Herausleiten des optischen Signals durch die Epidermis und eine äußere Vorrichtung zur Gewinnung des elektrischen Signals, wobei entweder optische Fasern eingesetzt oder die Schwächung des Lichtsignals durch die Absorption im Gewebe in Kauf genommen werden. Letzteres ist nicht störend, wenn die Fluoreszenzabklingzeit das glucoseabhängige optische Signal darstellt [32]. Als mechanische Messgrößen wurden anstelle der hier dargestellten Viskosität das glucoseabhängige Volumen eines sensorischen Gels [33] oder der glucoseabhängigen Druck in einer mit sensitiver Flüssigkeit gefüllten osmotischen Zelle $[34,35]$ genutzt. Für die Affinitätsviskosimetrie wurden neben dem bereits erwähnten minimalinvasiven Kapillarviskosimeter [12] relativ große elektromechanische Vorrichtungen mit rotierenden Magneten [36] und ein MEMS mit einem oszillierendem Kantilever und einer makroskopischen optischen Messvorrichtung [37] eingesetzt.

Bei dem von uns entwickelten viskosimetrischen Glucosesensor kommt erstmalig ein MEMS zum Einsatz, das die Bewegung der sensitiven Flüssigkeit erzeugt und gleichzeitig auswertet. Erstmalig ist es gelungen, das Mikroviskosimeter mit einer Mikrodialysekammer eines Volumens von nur ca. $100 \mathrm{nl}$ zu verbinden und die sensitive Flüssigkeit in dieser Kammer langzeitstabil einzuschließen. Der miniaturisierte Sensor besitzt eine hohe Genauigkeit und Stabilität des Mess-Signals in Elektrolytlösungen, die in ihrer lonenzusammensetzung der interstitiellen Flüssigkeit ähneln und ermöglicht genaue Glucosebestimmungen in vitro. Er steht vor der Erprobung in klinischen Studien und kann im Erfolgsfall für den Zeitraum von mehreren Tagen bis zu einer Woche zur kontinuierlichen Aufzeichnung der Glucosekonzentration von Probanden mit Diabetis mellitus eingesetzt werden. Sollte sich zeigen, dass das physiologische System die Genauigkeit der Glucosebestimmung mit diesem Sensor nicht beeinträchtigt, könnten auf der Grundlage der bisherigen Entwicklung Verfahren der Halbleitertechnologie und Mikrosystemtechnik zur industriellen Produktion eines minimalinvasiven Sensors für das subkutane Glucosemonitoring entwickelt werden.

Zusammenfassend kann festgestellt werden, dass mit dem von uns entwickelten System ein neuartiger Glucosemonitor zur Verfügung steht, der in Laboruntersuchungen driftfrei mit hoher Genauigkeit funktionierte. Gegenwärtig werden Sensorsysteme in einer Prototypen-Serie für den Einsatz in klinischen Studien aufgebaut. Sollte sich die in vitro gefundene Genauigkeit und Langzeitstabilität des Sensors auf in vivo Verhältnisse übertragen lassen, kann der Sensor künftig wirksam zur Verbesserung der Diabetestherapie beitragen. 


\section{Danksagung}

Die hier vorgestellten Entwicklungsarbeiten erfolgten im Rahmen BMBF- und BMWi-geförderter Projekte (Förderkennzeichen 0313862B und KF 0653901 UL8). Wir danken den Mitarbeitern der IHP-Pilotlinie und der Abteilung Process Research für die Chippräparation und die Durchführung der für die Mikromechanik erforderlichen Teilschrittentwicklungen sowie denen der Off-line Analytik für Charakterisierungsuntersuchungen. Darüber hinaus danken wir dem Aktionszentrum BioTOP und dem Diagnostik-Netzwerk Berlin-Brandenburg für die kontinuierliche Unterstützung des Projekts.

\section{Referenzen}

[1] Hönes J, Müller P. The technology behind glucose meters: test strips. Diab. Technol. \& Therap. 10 (2008) 10.

[2] Heinemann L. Clinical development of continuous glucose monitoring systems: considerations for the optimal strategy. Diab. Res. Clin. Prac. 74 (2006), 82.

[3] Davis S, Alonso MD. Hypoglycemia as a barrier to glycemic control. J. Diab. Complic. 18 (2004) 60.

[4] Vazeou A. Continuous blood glucose monitoring in diabetes treatment. Diab. Res. Clin. Prac. 93 (2011) 125.

[5] Klonoff DC. Continuous glucose monitoring. Roadmap for $21^{\text {st }}$ century diabetes therapy. Diab. Care 28 (2005) 1231.

[6] Francescato MP, Geat M, Stel G, Cauci S. Accuracy of a portable glucose meter and of a continuous glucose monitoring device. Clin. Chim. Acta 413 (2012) 312.

[7] Gough DA, Armour JC, Baker DA. Advances and prospects in glucose assay technology. Diabetol. 40 (1997) 102

[8] Schultz J, Mansouri S, Goldstein I. Affinity sensor: A new technique for the developing implantable sensors for glucose and other metabolites. Diabet. Care 5 (1982) 245.

[9] Ballerstädt R, Ehwald R. Suitability of aqueous dispersions of dextran and Concanavalin A for Glucose sensing in different variants of the affinity sensor. Biosens. \& Bioelectro. 9 (1994) 557.

[10] Ehwald R, Ballerstädt R, Dautzenberg H. Viscosimetric affinity assay. Analyt. Biochem. 234 (1996) 1.

[11] Beyer U, Ehwald R, Fleischer L-G. Post-stress thickening of dextran/concanavalin A solutions used as sensitive fluids in a viscosimetric affinity assay for glucose. Biotechnol. Prog. 13 (1997) 722.

[12] Beyer U, Schäfer D, Thomas A, Aulich H, Haueter U, Reihl B, Ehwald R. Recording of subcutaneous glucose dynamics by a viscosimetric affinity sensor. Diabetol. 44 (2001) 416.

[13] Diem P, Kalt L, Haueter U, Krinelke L, Fajfr R, Reihl B, Beyer U. Clinical performance of a continuous viscometric affinity sensor for glucose. Diab. Technol. \& Therap. 6 (2004) 790.

[14] Ehwald R. Mikrosensor zur Bestimmung der Konzentration von Glukose und anderen Analyten in Flüssigkeien auf der Basis der Affinitätsviskosimetrie. Patentschrift DE 19501159 B4 (2004).

[15] Ehwald K-E, Ehwald R, Knoll D, Winkler W, Zinke H. Vorrichtung und Verfahren zur Viskositätsmessung sowie Verfahren zur Herstellung einer Vorrichtung. Patentschrift DE 10027 684 B4 2005.09.01 (2005).

[16] Birkholz M, Ehwald K-E, Kulse P, Drews J, Fröhlich M, Haak U, Kaynak M, Matthus E, Schulz K, Wolansky D. Ultrathin TiN Membranes as a Technology Platform for CMOS-integrated MEMS and BioMEMS Devices. Adv. Func. Mat. 21 (2011) 1652.

[17] Birkholz M, Ehwald K-E, Ehwald R, Kaynak M, Borngräber J, Drews J, Haak U, Klatt J, Matthus E, Schoof G, Schulz K, Tillack B, Winkler W, Wolansky D. Mikroviskosimeter zur kontinuierlichen Glucosemessessung bei Diabetes mellitus. Mikrosystemtechnik-Kongress 2009, VDE-Verlag, Berlin (2009) 124.

[18] Birkholz M, Ehwald K-E, Wolansky D, Costina I, Baristiran-Kaynak C, Fröhlich M, Beyer H, Kapp A, Lisdat A. Corrosion-resistant metal layers from a CMOS process for bioelectronic applications. Surf. Coat. Technol. 204 (2010) 2055.

[19] Fröhlich M. Untersuchungen zur Biostabilität und Sterilisierbarkeit eines implantierbaren Glucosesensors, Diplomarbeit an der Universität Magdeburg (2011).

[20] Kulse P. Elektrische Charakterisierung von Mikroviskosimeterstrukturen. Diplomarbeit an der Fachhochschule für Technik und Wirtschaft Berlin (2009). 
[21] Kulse P, Birkholz M, Ehwald K-E, Bauer J, Drews J, Haak U, Höppner W, Katzer J, Schulz K, Wolansky D. Fabrication of MEMS actuators from the BEOL of a $0.25 \mu \mathrm{m}$ BiCMOS Technology Platform. Microelectr. Eng. (2012) submitted

[22] Drews J, Ehwald K-E, Schulz K. MEMS-Mikroviskosimeter und Verfahren zu seiner Herstellung. Patentschrift DE 102007031128 A1 (2009).

[23] Birkholz M, Ehwald K-E, Kaynak M, Semperowitsch T, Holz B, Nordhoff S. Separation of extremely miniaturized medical sensors by IR laser dicing. J. Optoelectr. Adv. Mat. 12 (2010) 479.

[24] Ehwald R, Ehwald M, Schoenfuss D, Szeponik J. Mikrodialysekammersystem mit eingeschlossenen kolloidalen Stoffen und Verfahren zu dessen Herstellung. Patentschrift WO 2009/144254 A1.

[25] Woehlecke H, Ehwald R. Characterization of size-permeation limits of cell walls and porousseparation materials by high-performance size-exclusion chromatography. J. Chromatogr. 708 (1995) 263.

[26] Erickson HP. Size of protein molecules at the nanometer level determined by sedimentation, gel filtration, and electron microscopy. Shulin $\mathrm{Li}$ (ed.) Biological procedures online 11/1, ULR Springer protocols.com, Springer links.com (2009) 32.

[27] Beyer U, Ehwald R. Compensation of temperature and Concanavalin A concentration effects for glucose determination by the viscosimetric affinity sensor. Biotechnol. Progr. 16 (2000) 1119.

[28] Garg SK. Glucose monitoring: an important tool for improving glucose control and reducing hypoglycemia. Diab. Technol. \& Therap. 10 (2008) 1.

[29] Li S, Davis EN, Anderson J, Lin Q, Wang Q. Development of boronic acid grafted random copolymer sensing fluid for continuous glucose monitoring. Biomacromol. 10 (2009) 113.

[30] Ballerstadt R, Evans C, Gowda A, McNichols R. In vivo performance evaluation of a transdermal near-infrared fluorescence resonance energy transfer affinity sensor for continuous glucose montoring. Diab. Technol. \& Therap. 8 (2006) 296.

[31] Ballerstadt R, Kholodnykh A, Colton E, Boretsky A, Motamedi M. Affinity-based turbidity sensor for glucose monitoring by optical coherence tomography: toward the development of an implantable sensor. Anal. Chem. 79 (2007) 6965.

[32] Nielsen JK, Christiansen JS, Kristensen SJ, Toft HO, Hansen LB, Soren A, Gregorius K. Clinical evaluation of a transcutaneous interrogated fluorescence lifetime-based microsensor for continuous glucose reading. J. Diab. Sci. Technol. 3 (2009) 98.

[33] Tierney S, Volden S, Stokke BT. Glucose sensors based on a responsive gel incorporated as Fabry-Perot cavity in a fiber-optic readout platform. Biosens. \& Bioelectron. 24 (2009) 2034.

[34] Krieftewirth M, Beyer U, Doss A, Ehwald R. Affinity sensor based on a membrane osmometer. Techn. Mess. 71 (2004), 29.

[35] Krushinitskaya $\mathrm{O}$, Tonessen $\mathrm{TI}$, Jacobsen $\mathrm{H}$, Johannessen EA. Characterization of nanoporous membranes for implementation in an osmotic glucose sensor based on the concanavalin A-dextran affinity assay. J. Membr. Sci. 376 (2011) 153.

[36] Boss C, Meurville E, Sallese J-M, Ryser P. Novel chemico-mechanical approach towards long-term implantable glucose sensing. Procedia Chem. 1 (2009) 313.

[37] Zhao Y, Li, S, Davidson A. A MEMS viscosimetric sensor for continuous glucose monitoring. J. Micromech. Microeng. 17 (2007) 2528. 